\title{
Reflets
}

Revue ontaroise d'intervention sociale et communautaire

\section{L'intégration des personnes ayant une incapacité, un avenir à créer}

\section{Roxanne Burelle}

Volume 4, numéro 2, automne 1998

Personnes vivant avec une incapacité

URI : https://id.erudit.org/iderudit/026216ar

DOI : https://doi.org/10.7202/026216ar

Aller au sommaire du numéro

Éditeur(s)

Reflets : Revue ontaroise d'intervention sociale et communautaire

ISSN

1203-4576 (imprimé)

1712-8498 (numérique)

Découvrir la revue

Citer cet article

Burelle, R. (1998). L'intégration des personnes ayant une incapacité, un avenir à créer. Reflets, 4(2), 32-46. https://doi.org/10.7202/026216ar

Tous droits réservés (C) Reflets : Revue ontaroise d'intervention sociale et communautaire, 1998
Ce document est protégé par la loi sur le droit d'auteur. L'utilisation des services d'Érudit (y compris la reproduction) est assujettie à sa politique d'utilisation que vous pouvez consulter en ligne.

https://apropos.erudit.org/fr/usagers/politique-dutilisation/ 


\title{
L'intégration des personnes ayant une incapacité, un avenir à créer
}

\author{
Roxanne Burelle
}

Analyste dans le domaine des ressources humaines, Ottawa

\section{Introduction}

\author{
"...le statut social \\ accordé aux personnes \\ ayant une incapacité \\ est dicté par les valeurs \\ et les croyances de la \\ majorité."
}

Les croyances et les mœurs sociaux sont des composantes intrinsèques aux acteurs vivant au sein d'une société. Ce sont également ces convictions qui dictent le modus operandi ou les pratiques usuelles au cours d'une période donnée. Or, celles-ci ne sont pas statiques, elles évoluent. Ce qui composait jadis l'imaginaire social d'un groupe d'acteurs ne reflète pas nécessairement celui des groupes sociaux actuels ou futurs. Il en est ainsi des croyances, des mœurs et des pratiques. La façon dont les acteurs perçoivent et gèrent les différences permet d'établir les balises de l'inclusion et de l'exclusion sociétales. Cela donne lieu à des affrontements continus et au dénouement incertain entre des acteurs, gardiens du statu quo, et ceux revendiquant des changements. C'est au terme de ce combat que se forgent les balises sociales et politiques des sociétés.

Résultats d'accidents, de maladies ou de malformations congénitales, les incapacités sont omniprésentes au sein de l'espèce humaine. Hormis ce fait, historiquement incontestable, le statut social accordé aux personnes ayant une incapacité est dicté par les valeurs et les croyances de la majorité. C'est dans cette perspective que nous avons élaboré les principaux thèmes de cet article.Ainsi, dans un premier temps, nous explorerons l'évolution 
et l'impact de la politique sociale, puis l'émergence de l'eugénisme, ses sources et ses conséquences. Dans un second temps, nous enchaînerons en analysant les principaux changements institutionnels et sociaux survenus à la suite de la proclamation, par les Nations Unies, de l'année internationale des personnes handicapées. Puis, nous ferons une brève incursion du côté des programmes et des politiques sociales actuellement en vigueur au Canada. Finalement, notre conclusion fera état de la situation socio-économique actuelle des Canadiens et Canadiennes ayant une incapacité.

\section{L'émergence de la politique sociale}

"L'exclusion sociale résulte de la marginalisation d'un groupe par un autre. La capacité d'exclure certains groupes d'individus au sein d'une société relève de forces intrinsèques aux acteurs eux-mêmes.»
Selon Patrick de Laubier (1984), l'apparition d'une politique proprement sociale au milieu du XIX ${ }^{\mathrm{e}}$ siècle ne relève pas du hasard. Elle est étroitement liée à la présence de trois facteurs au sein des sociétés européennes : l'industrialisation, l'émergence de l'emprise démocratique ainsi qu'une prise de conscience de droits politiques et sociaux (de Laubier 1984:7). L'industrialisation aurait occasionné une accumulation et une polarisation de la richesse entraînant ainsi un déracinement des populations rurales et une intensification de la misère (de Laubier 1984: 8). En réaction à cette paupérisation croissante des classes populaires et devant les dangers qu'elles pouvaient inspirer, les élites de l'époque ont proposé des mesures visant à changer ou à améliorer les conditions de vie matérielle et culturelle de ces classes. Cette prise de conscience progressive des droits sociaux pose les assises d'un discours et d'une politique proprement sociale.

Période de grands bouleversements, les programmes qui en résultent sont ceux de l'assurance-chômage, de la sécurité sociale ou des régimes de pension. Ils visent à garantir certaines dispositions fondamentales aux bénéficiaires. Malgré tout, les inégalités sociales (pauvreté, faim, inégalité salariale, etc.) subsistent et la situation socioéconomique des personnes vivant avec une incapacité demeure précaire. Or, comment faut-il expliquer, dans un contexte politique et social à visée égalitaire, la persistance de telles inégalités? 
"Ainsi, les croyances, moeurs et pratiques $d u$ groupe dominant au sein d'une société donnée dictent les paramètres de l'exclusion et l'inclusion sociale, de l'inacceptable et de l'acceptable. Imprégnés des valeurs, des préjugés et des préoccupations $d u$ groupe dominant qui les a élaborés et les a mis en ceuvre, les projets politiques, les lois et les programmes sociaux reflètent cet imaginaire social.»
L'exclusion sociale résulte de la marginalisation d'un groupe par un autre. La capacité d'exclure certains groupes d'individus au sein d'une société relève de forces intrinsèques aux acteurs euxmêmes. Selon Goffman (1963), influencés par leurs valeurs, leurs préjugés et leurs propres insécurités, les êtres humains tendent à marginaliser les groupes qui ne répondent pas à leurs idéaux ou leurs attentes. Ces exclus, qualifiés d' "anormaux», sont maintenus dans cet état de marginalisation au moyen de diverses formes de discrimination (Goffman 1963:15).

Il va de soi que, par définition, nous pensons qu'une personne ayant un stigmate n'est pas tout à fait humaine. Partant de ce postulat, nous pratiquons toutes sortes de discriminations, par lesquelles nous réduisons efficacement, bien que souvent inconsciemment, les chances de cette personne (Goffman 1963:15).

L'histoire des sociétés humaines comporte de nombreux exemples de discrimination (différences raciales, sexuelles, ethniques, politiques, religieuses, etc.).

Ainsi, les croyances, mœurs et pratiques du groupe dominant au sein d'une société donnée dictent les paramètres de l'exclusion et l'inclusion sociale, de l'inacceptable et de l'acceptable. Imprégnés des valeurs, des préjugés et des préoccupations du groupe dominant qui les a élaborés et les a mis en œuvre, les projets politiques, les lois et les programmes sociaux reflètent cet imaginaire social.

\section{L'eugénisme et les politiques d'institutionnalisation}

Le discours politique entourant l'intégration sociale des personnes ayant une incapacité prend diverses expressions au cours du XX siècle. Il s'inscrit dans un contexte sociétal caractérisé d'une part, par la création de grands projets à visée sociale et égalitaire et, d'autre part, par l'émergence de l'Eugénique scientifique issue du darwinisme. Alors que la politique sociale promeut l'égalité, 
l'eugénisme soutient le concept de supériorité d'un groupe visà-vis d'un autre (Rey 1995: 840). Ce sont là deux théories diamétralement opposées qui auront un impact indéniable sur les personnes vivant avec une incapacité.

On attribue à Francis Galton la paternité de l'Eugénique scientifique. Influencé par les théories de son cousin Darwin sur la sélection naturelle des espèces, Galton énonce les principes fondamentaux de cette nouvelle «science», soit: «entraver la multiplication des inaptes (...) et améliorer la race en favorisant la reproduction des plus aptes» (Rey 1995: 840). Peu à peu, le mouvement prend de l'essor et les principes s'insèrent dans le discours politique. L'impact du mouvement conduit à la création de lois permettant à l'État d'institutionnaliser et, dans certains cas, de justifier la stérilisation d'individus désignés inaptes.

Au Canada, la décennie qui précède la Seconde Guerre Mondiale marque l'apogée de l'Eugénique scientifique dans le discours politique. Appuyé par le corps médical, on construit des asiles et des hôpitaux qui serviront à isoler, entre autres, les individus «faibles de corps ou d'esprit». Nombre de personnes ayant une incapacité réussissent pourtant à échapper à ce sort; bien qu'elles demeurent socialement isolées et exclues. Cette pratique domine jusqu'à la fin des années cinquante. C'est dans la foulée des bouleversements sociaux des années soixante qu'un nouveau discours émerge (Statistique Canada 1991a: 150). Dès lors, les

"D’une part, l'état psychologique des personnes vivant la transition est intrinsèquement lié au phénomène: affaiblis par les années d'isolement, ces individus sont dépourvus des outils nécessaires pour vivre en communauté.» revendications des organismes de parents, inquiets des effets de l'isolement de leurs enfants, et des groupes de pression faisant la promotion des droits humains, forcent les États à questionner le bien-fondé de l'institutionnalisation (Statistique Canada 1991a: 150). Les années soixante marquent le début d'une ère de désinstitutionnalisation.

Durant les années 1960, le démantèlement des structures n'est toutefois pas sans conséquence. Il engendre des problèmes imprévisibles, notamment un taux d'errance élevé parmi les individus récemment désinstitutionnalisés. D'une part, l'état psychologique des personnes vivant la transition est intrinsèquement lié au phénomène: affaiblis par les années d'isolement, ces individus sont dépourvus des outils nécessaires 
pour vivre en communauté. D’autre part, dénuées de programmes ou mesures pouvant faciliter l'insertion de ces citoyens, les communautés font de leur mieux pour répondre à leurs besoins fondamentaux. Les autorités gouvernementales cautionnent généralement le principe d'intégrer ou de réintégrer les individus vivant avec une incapacité, mais elles ignorent les conséquences d'un tel projet (accessibilité des édifices, accessibilité au transport en commun, persistance des préjugés envers les personnes ayant une incapacité, etc.). Ainsi, en dépit des efforts déployés par les groupes de parents et d'autres associations, on tarde à remédier à la situation. Même en l'absence de mesures concrètes, cette période signale toutefois la fin de l'Eugénique scientifique et des politiques d'institutionnalisation et le début de l'intégration socioéconomique des personnes ayant une incapacité. Du moins, c'est l'idéal vers lequel on va tendre dorénavant.

\section{1, l'ère du renouveau}

"L'acte de présence ne suffit plus. Les individus ayant une incapacité veulent contribuer et participer activement et pleinement à la société."
Une proclamation allait relancer le débat de l'intégration sociale des personnes vivant avec une incapacité. En effet, les Nations Unies, en faisant de 1981 l'année internationale des personnes handicapées, propulsent à l'avant-scène internationale, leurs revendications sociales, concrétisent leur état de marginalisation, contribuent à promouvoir leurs droits et sensibilisent le monde entier aux difficultés que ces personnes surmontent quotidiennement. Cette proclamation symbolise une vision nouvelle du groupe. Dès lors, l'intégration des personnes ayant une incapacité s'insère concrètement dans le discours politique de nos sociétés et dans l'imaginaire social de ses citoyens. L'acte de présence ne suffit plus. Les individus ayant une incapacité veulent contribuer et participer activement et pleinement à la société.

Au Canada, cette proclamation a de multiples répercussions et un nombre considérable d'efforts sont déployés en vue de mettre sur pied des programmes et instaurer des nouvelles mesures. 
"Ainsi naît, en 1984, la Commission Abella. On doit à cette commission la définition de plusieurs termes liés à l'équité, dont celui d' 'équité en matière d'emploi». Expression heureuse, s'il en est, puisqu'elle englobe aujourd'hui l'ensemble des programmes conçus visant à remédier aux inégalités de chances vécues par certains groupes.»
Certaines de ces répercussions sont d'ordre juridique. L'article 15 paragraphe (1) de la Charte canadienne des droits et libertés assurant à tous le droit à la protection et au bénéfice de la loi indépendamment de toute discrimination est, en son principe, précisé par le paragraphe (2). Celui-ci vise à assurer la viabilité des activités, des lois et des programmes établis dans le but d'améliorer la situation des groupes spécifiés, au nombre desquels on trouve les personnes vivant une incapacité (Publications Canada 1982 : 15). Par ailleurs, on met sur pied des commissions d'enquête dont l'objectif est d'étudier l'état de la population handicapée canadienne et de recommander des mesures appropriées.

Ainsi naît, en 1984, la Commission Abella. On doit à cette commission la définition de plusieurs termes liés à l'équité, dont celui d' «équité en matière d'emploi». Expression heureuse, s'il en est, puisqu'elle englobe aujourd'hui l'ensemble des programmes conçus visant à remédier aux inégalités de chances vécues par certains groupes.Au départ, le mandat de la commission consistait à dresser le profil socio-économique de quatre groupes visés par la Loi sur l'équité: les femmes, les autochtones, les minorités visibles ainsi que les personnes ayant une incapacité (Moreau 1991 :26). Pour ce faire, la commission a examiné les données relatives au taux d'activité et de chômage, la prévalence de la ségrégation professionnelle ainsi que les revenus moyens de chacun des groupes ciblés, afin d'établir leurs profils socio-économiques. Les observations ont confirmé que les inégalités socio-économiques résultent non seulement des pratiques, croyances et préjugés inhérents aux individus d'une société donnée, mais également de facteurs intrinsèques aux structures politiques (Moreau 1991 :26). La Commission fit ses recommandations qui ont inspiré toute une série de programmes.

Au nombre de programmes développés par le gouvernement canadien, le programme d'action positive est instauré 1984. Sous la direction de la Commission canadienne des droits de la personne, ce programme vise à contrecarrer toute discrimination possible au sein des structures, en assurant le recrutement actif de personnes ayant une limitation fonctionnelle (Commission canadienne des droits de la personne Canada, sans date: 21). Il tente donc de 
procurer des opportunités de travail à des individus qualifiés mais chroniquement désavantagés ou systématiquement exclus du marché de l'emploi par des critères de sélection discriminatoires (Commission canadienne des droits de la personne Canada, s.d. : 21). En 1985, le programme d'équité en matière d'emploi lui succèdera et sera sous la gouverne de la Commission de la fonction publique fédérale.

La Loi sur l'équité en matière d'emploi étend la portée du discours de l'équité. En 1986, deux programmes liés à l'application de cette loi sont créés: le programme de contrats fédéraux et le programme législatif d'équité en matière d'emploi. Par eux, on cherche à éliminer les pratiques limitant l'accès au marché du travail pour les groupes visés et, ainsi, leur assurer un taux d'emploi proportionnel à leur représentation au sein de la population active.

Depuis 1988, le programme législatif d'équité en matière d'emploi oblige les employeurs ayant cent employés et plus, à soumettre des rapports annuels faisant état des données statistiques et à établir un plan d'équité où sont identifiés les obstacles à l'emploi et les moyens de les éliminer. Ce plan doit aussi favoriser l'adoption de politiques d'action positive en s'assurant qu'elles tiennent compte des différences (Statistique Canada 1993b : 49). Par ailleurs, le programme de contrats fédéraux soumet les entreprises de plus de cent employés ayant des ententes de biens et services avec le gouvernement fédéral, à des consignes précises. En effet, lors d'appels d'offre gouvernementale excédant 200,000 \$ ou plus, les entreprises soumissionnaires doivent s'engager à respecter les directives de la Loi sur l'équité en matière de l'emploi (Statistique Canada 1993b: 50). Un document dûment signé atteste l'engagement des entreprises et des preuves concrètes sont périodiquement requises et exigées d'elles (Statistique Canada $1993 \mathrm{~b}: 50)$.

En Ontario, à la même époque, le gouvernement provincial a créé l'Office des personnes handicapées. Les principes qui régissent cette agence gouvernementale reflètent d'emblée les valeurs dominantes, car les personnes ayant une incapacité y sont vues comme partie intégrante de la société canadienne et garantes des même droits que la population bien portante (Office des personnes 
«Manifestement, la création des

programmes et la mise

en vigueur de

politiques portent à

croire que le processus

d'intégration des

personnes ayant une

limitation fonctionnelle est formellement

engagé.» handicapées $1988: 1)$. On constate également qu'elles sont, avec les organismes qui défendent leurs intérêts, les plus aptes à déterminer leurs besoins et à suggérer des recommandations quant aux services disponibles et aux programmes existants (Office des personnes handicapées $1988:$ i).

Manifestement, la création des programmes et la mise en vigueur de politiques portent à croire que le processus d'intégration des personnes ayant une limitation fonctionnelle est formellement engagé. Or, qu'en est-il réellement? Le discours politique et juridique ont certes évolué en direction d'une plus grande égalité, mais ont-ils eu l'impact désiré parmi les groupes ciblés? Les lois et programmes ont-ils réussi à réparer les torts du passé? Ce que l'on constate, c'est que la période des années 1980, avec son discours qui promeut l'intégration socio-économique des personnes vivant une incapacité, offre une vision diamétralement opposée à celle du début de siècle. Peut-on dire que les structures et la perception sociales qui prévalent en font autant? Seul un examen de la situation socio-économique de ces individus peut le confirmer.

\section{La situation de classe}

MaxWeber (1971) définit la probabilité qu'une population donnée parvienne à certains objectifs ou aient des chances dans la vie comparables à celles d'autres groupes comme étant la situation de classe. Il soutient que celle-ci dépend de la capacité du groupe de s'accaparer et de disposer des services et des biens afin de se procurer des rentes ou des revenus (Weber 1971: 309). Parallèlement, elle relève son aptitude à contrôler les biens et les compétences qui résultent de leur usage économique dans un système donné (Weber 1971: 309). Le concept de situation de classes de Max Weber se veut ainsi intrinsèquement lié à la capacité du groupe de participer activement à l'économie d'une société, voire de s'intégrer socialement et économiquement à cette dernière. Ainsi, on peut mesurer l'impact des mesures instituées 
«On estime à 12, $7 \%$ $(2,297,135$ millions de Canadiennes et Canadiens), le nombre d'individus entre 15 et 64 ans vivant avec une incapacité... Cette population comprend des individus ayant des déficiences auditives, mentales, visuelles, physiques, intellectuelles et des troubles d'apprentissages.» par les gouvernements en examinant les effets qu'elles ont sur la participation socio-économique des groupes ciblés.

Dans cette optique, l'Enquête sur la santé et les limitations d'activités s'avère une source précieuse d'information. Elle permet de mesurer, entre autres, les taux de fréquentation scolaire, les taux d'activité et d'inactivité ainsi que les niveaux de pauvreté propres aux personnes vivant avec une incapacité.

On estime à 12,7 \% (2,297,135 millions de Canadiennes et Canadiens), le nombre d'individus entre 15 et 64 ans vivant avec une incapacité (Conseil de recherches en sciences humaines du Canada 1993 : 1). Cette population comprend des individus ayant des déficiences auditives, mentales, visuelles, physiques, intellectuelles et des troubles d'apprentissages. Une population aussi diverse complique quelque peu la tâche de déterminer les effets qu'ont eu les mesures prises durant les années 1980. Aussi, convient-il de préciser que le profil qu'on dressera de ces sousgroupes se veut d'abord et avant tout général.

\section{La formation scolaire}

Plusieurs études ont démontré l'existence d'une corrélation positive entre le niveau de scolarité atteint par un individu et son revenu. Pour les personnes ayant une incapacité, ce lien a des conséquences bien plus grandes que celles vécues par les individus bien-portants, car elles fréquentent proportionnellement moins les établissements scolaires. Les statistiques démontrent que $29 \%$ des individus ayant une limitation fonctionnelle cumulent moins de neuf années de scolarité alors que ce taux se situe à $11 \% \mathrm{chez}$ les individus bien-portants (Nessner 1990b: 5). De même, respectivement $39,9 \%$ et $26 \%$ des individus ayant une limitation fonctionnelle détiennent un diplôme d'études secondaires ou ont entrepris des études post-secondaires, comparativement à 44,3\% et $33,7 \%$ pour l'ensemble de la population canadienne (Statistique Canada 1990a: 26). Cet écart se reflète également dans la 
«Or, en 1991, il y

avait 232,125

enfants ayant une

limitation

fonctionnelle qui ne

bénéficiaient toujours

pas de services

fondamentaux comme

ceux d'un preneur de

note, d'un

accompagnateur ou

d'un lecteur leur

permettant de

s'intégrer et participer

pleinement à

l'expérience scolaire." proportion des individus âgés de 25 à 34 ans qui affirment détenir un diplôme d'études universitaires. En effet, on chiffre à $7 \%$ le taux de diplômés universitaires chez les personnes ayant une limitation fonctionnelle, alors que ce taux est de $14 \%$ dans l'ensemble de la population canadienne (Statistique Canada 1990c : 26). Cela dit, les incapacités peuvent-elles rendre compte à elles seules de cet écart?

Certes, on ne peut pas nier l'impact qu'ont les troubles d'apprentissage, les déficiences physiques ou les problèmes psychiatriques sur la fréquentation scolaire des individus ayant une incapacité. Pourtant, les enquêtes démontrent qu'il existe aussi d'autres facteurs. L'inaccessibilité des lieux de formation explique l'écart entre les taux de fréquentation scolaire des individus ayant une incapacité et leurs collègues bien-portants. Les obstacles environnementaux sont de divers ordres. Pour un individu en fauteuil roulant ou éprouvant des problèmes de mobilité, certains éléments sont essentiels : ascenseurs permettant d'accéder aux étages supérieurs d'un édifice, espace suffisant entre les portes, rampes d'accès, barres d'appui, dénivellations aux passages piétonniers, etc. Pour les personnes ayant une déficience visuelle, la disponibilité d'aides techniques comme les lecteurs de braille, la présence de consignes rédigées en braille ou en gros caractères et les signaux sonores aux feux de circulation sont indispensables. Or, en 1991, il y avait 232,125 enfants ayant une limitation fonctionnelle qui ne bénéficiaient toujours pas de services fondamentaux comme ceux d'un preneur de note, d'un accompagnateur ou d'un lecteur leur permettant de s'intégrer et participer pleinement à l'expérience scolaire (Statistique Canada 1994 : 214).

La fréquentation scolaire des individus qu'ils aient ou non une incapacité dépend également de la disponibilité d'un mode de transport. À cet effet, il faut noter que nombre de communautés canadiennes ne disposent pas à ce jour d'un service de transport en commun pouvant accommoder les personnes ayant une incapacité. Au Canada, 11,750 enfants âgés de 5 à 14 ans doivent avoir recours à un véhicule adapté pour leurs déplacements locaux, alors que seulement $56 \%$ d'entre eux ont accès à un service de 
ce genre (Statistique Canada 1990a: viii). Sans un tel service, plusieurs enfants sont obligés de quitter leur communauté pour fréquenter un établissement scolaire (Statistique Canada 1994 : 212). Cette rupture avec le milieu familial est d'autant plus grande que, pour $82 \%$ de la population ayant une incapacité, la famille fournit un secours inestimable dans l'accomplissement de tâches quotidiennes (Statistique Canada 1990a : xi).

\section{Le taux d'activité}

Toutes proportions gardées, le taux d'activité des personnes ayant une incapacité, tout comme le taux de fréquentation scolaire, sont inférieurs à ceux de la population bien-portante. En 1986, $48,2 \%$ des personnes vivant avec une incapacité entre 15 et 34 ans déclarent occuper un emploi comparativement à $64,1 \% \mathrm{chez}$ les bien-portants (Statistique Canada 1988 : 26). Plus de 52,7\% des 15 à 24 ans et $65,8 \%$ des 25 à 34 ans affirment faire partie de la main-d'œuvre active (Statistique Canada 1990c: 28). Néanmoins, le taux d'inactivité relativement élevé du groupe cache une réalité quelque peu troublante. Plus de $20 \%$ des individus dits inactifs déclarent, au moment de l'enquête, être partiellement limités au travail (Statistique Canada 1994 : 16). Par ailleurs, près de $24 \%$ d'entre eux affirment qu'ils accepteraient un emploi à temps plein si pareille occasion se présentait (Statistique Canada 1994 : XII). Ces données portent à croire que l'inactivité du groupe ne résulte pas simplement de l'incapacité, mais dépend également de d'autres facteurs.

Le taux d'inactivité des personnes ayant une incapacité, tout comme le taux de fréquentation scolaire, sont intrinsèquement liés à la présence d'obstacles environnementaux. Ainsi, plus de $30 \%$ des individus dits inactifs déclarent ne pas pouvoir suivre de cours de formation professionnelle qui leur permettraient de réintégrer le marché de l'emploi, dû à l'inaccessibilité des établissements scolaires (Statistique Canada 1994 : 18). Près de $15,3 \%$ d'entre eux notent également l'absence d'un mode de 
transport adéquat et $51 \%$ déclarent que les frais de scolarité constituent une entrave sérieuse à l'atteinte de cet objectif (Statistique Canada 1994 : 18).

\section{Les obstacles systémiques}

«En effet, au terme d'une enquête, plus de $20 \%$ des individus ayant une incapacité affirment ne pas être activement à la recherche d'un emploi par crainte de devenir inadmissible à un programme de soutien du revenu (prestations d'aide sociale, prestations familiales ou une pension d'invalidité) ou à des programmes d'aide et services (assurancemédicaments, logement adapté).»
La situation de classe des individus ayant une incapacité est influencée par deux facteurs : leur état physique et les obstacles environnementaux. Or, ce ne sont pourtant pas les seuls. Des entraves d'ordre systémique existent. Les programmes institués sous l'égide de la politique sociale en matière d'équité ont leur lot d'effets pervers, celui de dissuader la poursuite d'activités telles que la recherche d'un emploi. En effet, au terme d'une enquête, plus de $20 \%$ des individus ayant une incapacité affirment ne pas être activement à la recherche d'un emploi par crainte de devenir inadmissible à un programme de soutien du revenu (prestations d'aide sociale, prestations familiales ou une pension d'invalidité) ou à des programmes d'aide et services (assurance-médicaments, logement adapté) (Statistique Canada 1993a : 52). Le manque de souplesse dans l'administration de ces programmes engendre de sérieuses conséquences. Il crée un état de dépendance économique et freine le processus d'intégration socio-économique des personnes ayant une incapacité. Parallèlement, il les maintient sous le joug de la pauvreté.

Au Canada, en 1986, on chiffre à plus de $28 \%$ le nombre d'individus ayant une incapacité qui vit, au sein d'une famille à faible revenu (Statistique Canada 1990c :31). Ce taux est d'autant plus frappant si on le compare à la proportion de familles à faible revenu au sein de la population bien-portante qui est de $14 \%$ (Statistique Canada 1990c : 31). Plus de $56 \%$ des personnes vivant avec une incapacité déclarent avoir un revenu inférieur à $10000 \$$ (Statistique Canada 1990c: XI). L'introduction de mesures telles que les crédits d'impôts et les déductions ne semblent pas remédier aux problèmes. En effet, les crédits non remboursables ont un effet négligeable sur la situation économique des individus ayant 
une limitation fonctionnelle, car la faiblesse de leurs revenus ne leur permet pas de bénéficier pleinement des avantages fiscaux dans le système actuel de taxation (Torjman 1988, 71). De toute évidence, l'établissement de telles mesures n'a pas tenu compte de la réalité socio-économique de la population concernée. Seule une réforme de la fiscalité, voire la transformation des crédits non remboursables en crédits remboursables, aurait pallié cette situation (Institut G. Allan Roeher 1991 : III).

\section{Conclusion}

"La lutte qu'on mène n'est plus dirigée principalement contre l'exclusion, mais pour l'intégration."
Aujourd'hui, pour avoir des chances égales d'accès sur le marché de l'emploi, le combat des personnes ayant une incapacité se livre sur un terrain où les barrières environnementales et la discrimination systémique constituent des obstacles de taille. La lutte qu'on mène n'est plus dirigée principalement contre l'exclusion, mais pour l'intégration. Toutes proportions gardées, les personnes ayant une incapacité sont moins scolarisées que leurs compatriotes bien-portants, participent moins activement au marché de l'emploi et font plus fréquemment partie d'une famille à faible revenu.

Malgré tout, il convient de noter que des progrès ont été faits. Depuis les années 1980, la situation de classe du groupe ne reflète en rien celle du début du siècle ou même celle des années 1960. Toutefois, comment peut-on expliquer que de tels écarts subsistent entre les deux groupes? Lors de l'élaboration et la mise en œuvre des mesures correctrices, l'ignorance concernant les obstacles environnementaux et systémiques est en partie responsable de ces écarts.

Cette omission a comme effet de nuancer certaines des mesures instaurées. Pourtant, on ne pense pas que cet oubli soit volontaire et conscient. Comme nous l'avions souligné au début de cet article, l'élaboration et la mise en œuvre de lois et de programmes demeurent intrinsèquement liés à l'imaginaire social des acteurs impliqués dans le processus. De même que les politiques instituées par le passé étaient le fruit de la réflexion d'acteurs bien-portants, 
"Pour être efficaces, les lois et programmes doivent être élaborés en fonction de la réalité de la population ciblée par des acteurs conscientisés à leurs vécus.» de même on ne peut être surpris par l'inadéquation de certaines politiques actuelles, car elles émergent de l'imaginaire du même groupe d'individus. Pour être efficaces, les lois et les programmes doivent être élaborés en fonction de la réalité de la population ciblée par des acteurs conscientisés à leurs vécus. Or, les personnes ayant une incapacité commencent peu à peu à s'impliquer dans le processus d'élaboration de politiques les concernant. Par ailleurs, leur perception et leur vision de leurs besoins acquièrent depuis peu une plus grande légitimité parmi la population bien-portante. Le rapport Scott en fait état. La réceptivité du milieu et l'absence d'obstacles environnementaux contribueront fortement à l'efficacité des mesures mises en place jusqu'à présent. Or, faut-il le rappeler, l'absence de services au sein de communautés canadiennes ne permet pas aux personnes ayant une incapacité de s'intégrer pleinement. Et, si l'intégration socio-économique des personnes ayant une déficience est l'objectif à atteindre, on doit par tous les moyens chercher à éliminer les obstacles environnementaux et systémiques. L'objectif sera atteint le jour où les personnes ayant une incapacité participeront socialement, politiquement et économiquement à la société canadienne.

\section{Bibliographie}

BOURDIEU, PIERRE (1992). Réponses, Paris, Éditions du Seuil.

BURELLE, ROXANNE (1995). L'intégration des personnes handicapées ou le voile de l'exclusion, thèse de maîtrise, Ottawa, Université d'Ottawa.

COMMISSION ABELLA (1984). Rapport de la commission sur l'égalité en matière d'emploi, Ottawa, Ministère des approvisionnements et services Canada.

COMMISSION CANADIENNE DES DROITS DE LA PERSONNE (sans date). Employ-Ability A Resource Guide, Ottawa, Santé et bien-être Canada.

COMMISSION DES DROITS DE LA PERSONNE DU QUÉBEC (1981). Droits et libertés, vol. 4 , no. 1.

CONSEIL DE RECHERCHES EN SCIENCES HUMAINES DU CANADA (1993). The Integration of People with Disabilities, Ottawa, Ministère des approvisionnements et services Canada.

DE LAUBIER, PATRICK(1984). La politique sociale dans les sociétés industrielles - 1800 à nos jours, Paris, Éditions Economica.

FAWCETT, GAIL (1996). Vivre avec une incapacité au Canada: un portrait économique, Ottawa, Ministère des approvisionnements et services Canada.

FREUND, JULIEN (1966). Sociologie de Max Weber, Paris, Presses universitaires de France.

GOFFMAN, ERVING (1975). Stigmate. Les usages sociaux des handicaps, Paris, Éditions de minuit. 
INSTITUT G. ALLAN ROEHER (1991). Pas de refuge dans le besoin, North York, Institut G. Allan Roeher.

MOREAU,JOANNE (1991). «L'équité en matière d'emploi», Tendances sociales canadiennes, Ottawa, Statistique Canada - Ministère des approvisionnements et services Canada, 26-30.

NESSNER, KATHERINE (1990a). «Les enfants ayant une incapacité». Tendances sociales canadiennes, Ottawa, Statistique Canada - Ministère des approvisionnements et services Canada, no. 19, 1820.

NESSNER, KATHERINE (1990b). «Profil des Canadiens ayant une incapacité». Tendances sociales canadiennes, Ottawa, Statistique Canada - Ministère des approvisionnements et services Canada, no. $18,2-5$.

PUBLICATIONS CANADA (1982). La Charte des droits et libertés: guide à l'intention des Canadiens, Ottawa, Ministère des approvisionnements et services Canada.

REY,ALAIN ET JOSETTE REY-DEBOVE (1995). Le Petit Robert, Montréal, Éditions DicoRobert. ROEHER INSTITUTE (1993). Direct Dollars A Study of Indiviualized Funding in Canada, North York, York University - Roeher Institute.

SANTÉ ET BIEN-ÊTRE SOCIAL CANADA (1980). Les personnes handicapées au Canada, Ottawa, Ministère des approvisionnements et services Canada.

STATISTIQUE CANADA (1994). Caractéristiques choisies des personnes ayant une incapacité, Ottawa, Ministère de l'industrie, des sciences et de la technologie.

STATISTIQUE CANADA (1993a). Caractéristiques de l'emploi et du niveau de scolarité chez le adultes ayant une incapacité, Ottawa. Ministère de l'industrie, des sciences et de la technologie.

STATISTIQUE CANADA (1993b). «Définition et mesure de l'équité en matière d'emploi». Perspectives, Ottawa, Ministère des approvisionnements et services Canada, hiver 1993b, 49-61.

STATISTIQUE CANADA (1991a). Entraide collective - Acte du symposium sur les soutiens sociaux, Ottawa, Ministère des approvisionnements et services Canada.

STATISTIQUE CANADA (1991b). Loisirs et habitudes de vie des personnes ayant une incapacité au Canada, Ottawa, Ministère des approvisionnements et services Canada.

STATISTIQUE CANADA (1990a). Enquête sur la santé et les limitations d'activités - Personnes ayant une incapacité au Canada, Ottawa, Ministère des approvisionnements et services Canada.

STATISTIQUE CANADA (1990b). Certaines conséquences socio-économiques de l'incapacité pour les femmes au Canada, Ottawa, Ministère des approvisionnements et services Canada.

STATISTIQUE CANADA (1990c). Recensement - Un profil des personnes ayant une incapacité au Canada, Ottawa, Ministère des approvisionnements et services Canada.

STATISTIQUE CANADA (1988). Activité sur le marché du travail des Canadiens atteints d'une incapacité, Ottawa, Ministère des approvisionnements et services Canada.

TORJMAN, SHERRI RESIN (1988). Le revenu précaire: le système de pensions d'invalidité au Canada, Downsview, Institut G. Allan Roeher.

WEBER, MAX (1971). Économie et société, Paris, Librairie Plon.

XIBERRAS, MARTINE (1993). Les théories de l'exclusion, Paris, Méridiens Klincksieck.

YUKER, HAROLD E. (1988). Attitudes Towards Persons with Disabilities, New-York, Springer Publishing Company. 